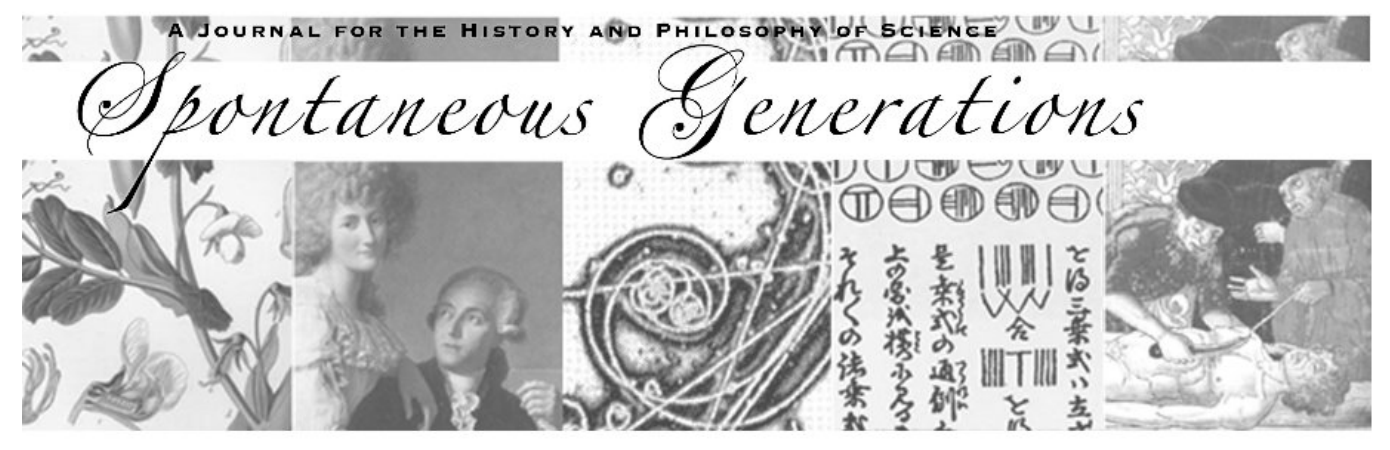

\title{
Progress in Science and Science at the Non-Western Peripheries
}

Author(s): Deepanwita Dasgupta

Source: Spontaneous Generations: A Journal for the History and Philosophy of Science, Vol. 3, No. 1 (2009) 142-157.

Published by: The University of Toronto

DOI: $10.4245 /$ sponge.v3i1.6575

EDITORIAL OFFICES

Institute for the History and Philosophy of Science and Technology

Room 316 Victoria College, 91 Charles Street West

Toronto, Ontario, Canada M5S 1K7

hapsat.society@utoronto.ca

Published online at jps.library.utoronto.ca/index.php/SpontaneousGenerations ISSN 19130465

Founded in 2006, Spontaneous Generations is an online academic journal published by graduate students at the Institute for the History and Philosophy of Science and Technology, University of Toronto. There is no subscription or membership fee. Spontaneous Generations provides immediate open access to its content on the principle that making research freely available to the public supports a greater global exchange of knowledge. 


\title{
Peer-Reviewed
}

\section{Progress in Science and Science at the Non-Western Peripheries*}

\author{
Deepanwita Dasgupta ${ }^{\dagger}$
}

\begin{abstract}
Assuming that progress in science means effectiveness at problem-solving, this paper discusses how a progressive scientific tradition can be created by a peripheral scientific community. A mechanism of peripheral scientific growth is proposed, and it is illustrated with an Indian case study. The conclusion of the paper is that scientific collaboration between metropolitan and peripheral research communities is frequently characterized by a persistent inequality of intellectual authority due to inequality in their epistemic transactions.
\end{abstract}

\section{INTRODUCTION}

The emergence of a scientific community at a given location depends on two things: first, the availability of a variety of theories and opinions to its members, and second, the ability of (some) members of that community to create new exemplars with the help of those theories. In Against Method (1988), Feyerabend argues that the smooth functioning of a progressive scientific community depends on two principles: first, the principle of tenacity, according to which scientists hold onto attractive theories in spite of initial difficulties so as to develop those theories' full potential, and second, the principle of proliferation, which constantly motivates scientists to produce new theories and perspectives, providing a rich marketplace of ideas. The meshing together of these two principles (within a research community) creates an autonomous and a progressive tradition in science. ${ }^{1}$

The debate on scientific knowledge has always focused most strongly upon the question of whether science is rational. But an equally

*Received July 2009. Revised paper accepted November 2009.

${ }^{\dagger} \mathrm{A}$ PhD candidate at the University of Minnesota, Deepanwita Dasgupta's research interests are knowledge-networks in science, science in the non-West, and divisions of cognitive labor in science.

${ }^{1}$ There is, of course, another crucial causal factor in the emergence of a new scientific community, i.e., the availability of sufficient funding. From the perspective of science policy, sufficient funding must be available to a scientific community before it can develop or pursue any research programs. My thanks to my anonymous referees for underscoring this point. 
controversial question has also been whether science is progressive, and if so, in exactly what sense science makes progress. Traditionally speaking, philosophers have always taken science to be a progressive enterprise (see for example, Reichenbach 1938; Popper 1963; Lakatos 1970; Laudan 1977; and more recently, Niiniluoto 1984; Kitcher 1993) but neither philosophers nor scientists have been able to answer, to everyone's satisfaction, what constitute the markers of such scientific progress.

Yet in a clearly intuitive sense, science does make progress, for we observe scientific communities solving numerous theoretical and practical problems. Talking about science-in the last resort-amounts to talking about such communities and their successful endeavours. This was a major insight offered by Kuhn in The Structure of Scientific Revolutions (1962) which I shall accept in this paper, and consider, following Kuhn, science to be the collective endeavours of such communities. For the purpose of this paper, I will take progress in science to mean how (some) scientific communities successfully carry out a particular piece of research in order to solve a problem, and how they communicate this result to their peers with the aim of receiving their assent and their consensus. ${ }^{2}$ Naturally, during this process of creation and communication of scientific knowledge, those communities are characterized by certain features that influence their epistemic endeavours and determine the outcome of their work. Progress in science can be construed in terms of the properties that determine the success (or, conversely, the failure) of their efforts, progress being achieved by those communities that exhibit some kind of epistemic gain.

The traditional literature on progress in science sees science mainly as a collection of theories, and thus, progress in science was viewed mainly as changes in the truth-contents of those theories. ${ }^{3}$ In 1977, however, Larry Laudan proposed an alternative reading of scientific progress (Laudan 1977). Instead of emphasizing the truth contents of a scientific theory, Laudan focused on effectiveness at problem-solving as the true marker of scientific activity. Thus, an increase in scientific knowledge means increased ability at problem-solving-whether this ability belongs to science in general or to a given scientific community. This was previously also said by Kuhn, and it is this approach that I shall accept for the purpose of this paper. Hence, instead of talking about scientific progress from

\footnotetext{
${ }^{2}$ Thus, in this essay progress will mean both an achievement and the process by which the community gains such achievements.

${ }^{3}$ However, important advances in science could be made when a scientific community discovers that the theory or the model that it has been entertaining is false. The importance of falsification in science has been underscored by both Popper and Lakatos, for avoiding errors is an important part of scientific practice. My thanks to my two anonymous referees for drawing my attention to this important point.
} 
a meta-level, in this paper I shall be concerned with scientific progress of particular scientific communities-how they produce a specific output of scientific research; how they engage in an inter-community dynamic in order to communicate their results to their peers; and how they gain a consensus. In short, I shall adopt in this paper a community-based, internalist view of scientific progress. ${ }^{4}$ Thus, the question of scientific progress will translate into a question of how scientific communities successfully conduct (and conclude) their research endeavours in order to create exemplars that give themselves the maximal problem-solving capacity and allow them to build a stable research tradition.

In this paper, I shall address the question of progressiveness of research communities from a non-standard angle. Instead of talking about scientific progress within the standard contexts of Euro-American research communities, I shall ask instead what makes a peripheral scientific community, i.e., a scientific community outside Europe and North America, progressive and how such research communities generate an output of scientific knowledge. I shall propose a mechanism of how scientific knowledge is made by such peripheral research communities-creating a condition of progressiveness for their communities at home-but also makes them crucially dependent upon science done elsewhere. To illustrate this theoretical framework of scientific knowledge-making under peripheral conditions, I shall introduce a case study from twentieth-century history of science-the formulation of Bose-Einstein statistics in 1924 by Satyendra Nath Bose, an unknown, young scientist from colonial British India. I shall argue that an analysis of Bose's work shows us the constraints of a peripheral scientific researcher (as well as that of his or her community, especially during its pioneering stages), revealing the stages through which the work of a peripheral researcher must pass before it can splice with the metropolitan scientific efforts so as to become part of normal science. But before we proceed to such theoretical considerations of scientific knowledge-making from the peripheries, let us first consider what makes a research community peripheral, what the epistemic markers

\footnotetext{
${ }^{4}$ For a lucid discussion on the different notion of progress implied in science, see Alexander Bird, "What is Scientific Progress?" Nous 41:1 (2007): 92-117. Bird discusses three possible ways to understand scientific progresses: as an accumulation of knowledge (epistemic progress); as an accumulation of truth or truth-likeness (semantic progress); and finally, as the enhancement of the problem-solving capacities of a research community (instrumental progress). In this essay, I shall adopt the perspective of instrumental progress, thereby assuming the standpoint of a researcher who is seeking to produce scientific knowledge in collaboration with his or her peers. Thus this essay will consider only under what circumstances such scientists would agree that they are making progress. My thanks to my two anonymous referees who urged me to clarify this important issue.
} 
of such peripherality are, and how it affects that community's ability to produce new scientific knowledge.

\section{What is Peripheral Science? Six Criteria of a Viable SCIENTIFIC COMMUNITY}

Research communities that are progressive in the sense of being more productive at problem-solving can exist in two contexts. Firstly, they may exist where people and their knowledge are highly concentrated, e.g., as in metropolitan communities. But a large number of scientific communities also work from various places of lesser knowledge concentration, from the so-called peripheries of scientific knowledge. Such communities generally work in an atmosphere of social and institutional isolation, away from the metropolitan centres of research, but nevertheless participate in the same task of making scientific knowledge. They are also often geographically distant from such centres, but physical distance alone is not the true marker of their peripherality. The complexity of modern science frequently distributes the cognitive labour of research among different cultures and societies, spreading scientific research among different research communities, but such globalizing trends alone do not make a community peripheral. The crucial marks of peripherality arise from an inequality of epistemic relationship between the two communities-when one cannot make knowledge or generate a consensus without involving the other.

Historically, we observe the emergence of such peripheral scientific communities from the late nineteenth century, when, in the wake of European expansion in Asia (and Africa), a number of peripheral research communities came into existence-consisting at first of many expatriate scientists and later of scientists of non-Western origin-in distant countries such as Japan, Korea, and India. Scientists from those distant centres have since then made significant contributions to scientific knowledge, but their internal dynamics of scientific knowledge-production and their interactions with their metropolitan peers have not yet received sufficient philosophical analysis and attention (but see Traweek 1988). The purpose of this paper is to begin tentatively this kind of work.

A question may be raised at this point about the aim of my paper-do I seek to provide a sociological or a philosophical account of non-Western science? While I think that a good sociological analysis may, in itself, provide us with important philosophical insights, what I shall try to develop in what follows is an epistemic analysis of peripherality-what is to be a peripheral researcher in science. The objective of this paper will therefore be to consider how a network of expertise and scientific knowledge is built in the peripheral context and how this network allows a peripheral research community to gain its first foothold in scientific research. This 
means, furthermore, that I shall be mostly concerned in this essay with the pioneering stages of a peripheral scientific community, i.e., with the time when such a community lacks a critical mass at home.

Thus, from the peripheral point of view, research communities can be divided into two sorts: first, the metropolitan communities, located at highly concentrated centres of scientific research, mostly located in different Euro-American centres, and a wider, sparser network of smaller research communities, located over a wider area of the globe. The landscape of scientific knowledge is thus not uniform-as is commonly presupposed-but rather uneven in nature. ${ }^{5}$ The researchers of the latter type remain socially and physically distant from their metropolitan communities-and their invisible colleges-but they share the same work of making scientific knowledge and thus naturally aspire to be counted among the progressive scientific communities. But their distance-whether social, geographical or institutional-places them under various constraints of peripherality, influencing the outcome of their research work. Given that this was the epistemic landscape within which much of the late nineteenth- and early twentieth-century science actually developed, a general analysis of the knowledge-making conditions of such scientific communities seems to be at least called for. The question thus arises: how do such communities work towards a condition of progressiveness that creates an autonomous research tradition at their home base? By what methods are their scientific outputs validated, how do they collaborate with their metropolitan peers, and could such peripheral communities ever develop a new paradigm in science? In the rest of this paper, I shall try to provide some tentative replies to these questions with the help of a simple feedback mechanism and a case study drawn from the peripheral location of India.

Little philosophical literature currently exists on how peripheral communities emerge, communicate, and carry on their task of making scientific knowledge, and what-if anything-keeps them from their goal of attaining scientific progress. Discussions of rationality and progressiveness in science are carried on primarily by keeping the metropolitan centres of science in view. The emergence of new research communities has, however, received some attention from a different type of analysis of scientific knowledge: analysis of science development and

\footnotetext{
${ }^{5}$ The distribution of scientific research in the world is presently more asymmetrical than the distribution of wealth. Research efforts and research funding are concentrated in a few Euro-American countries, which produce most of the journal literature in science and thus naturally dominate the reward structure of science. For a detailed discussion of this issue and of how peripheral scientific communities should respond to it, see Subbiah Arunachalam, "Peripherality in Science: What Should be Done to Help Peripheral Science Get Assimilated into Mainstream Science," Technical Report, Publication and Information Directorate, New Delhi, (1982): 67-76.
} 
science policy. In a science policy paper that focused on the question of what necessary elements must be in place before a new scientific community can emerge in a Less Developed Country (LDC) and what characteristics have to be exhibited by those newly emerging LDC communities, Mike Moravcsik, who spent considerable time as a science policy advisor in Pakistan, proposed an interesting list of six criteria (Moravcsik 1978, 8-13). This list of criteria determines, according to him, how a viable scientific community can emerge at a LDC location. Those proposed six parameters for the birth of a new community are as follows: 1) motivation, which includes curiosity, aesthetic satisfaction, or sense of collective achievement such as national glory; 2) subject matter, which, according to Moravcsik, is universal, no matter what the social context of the science in question; ${ }^{6} 3$ ) ideology, which implies that LCD scientists should be motivated, somehow, by the goals of possible international collaboration; ${ }^{7}$ 4) methodology, which is universal; and finally, the two criteria that link science to the issues of development: 5) applications and 6) infrastructure.

This interesting list provides good advice, no doubt, but it also shows why a newly emerging scientific community often finds itself heavily dependent upon science done elsewhere. In many non-Western locations such as India, modern science and the beginning of a professional scientific community typically begins with a phase of "colonial science," i.e., science organized to serve the needs of information for a colonial state. Most such locations began first with an array of field sciences, such as botany or geology, and only later on went to develop pure university-based sciences, such as physics. In scenarios with this kind of complication, the so-called uniform ingredients of scientific activity, such as methodology or subject matter, appeared, in the beginning, as large-scale imports from outside via a state-sponsored education system that introduced Western knowledge into the colonies (also known as the "civilizing mission"). A culture of scientific research in such locations is thus either perceived as an import, or worse, an imposition, having little root in the psyche of the indigenous researchers. As for the application and the infrastructure, the peripheral practitioners of science rarely inherit any functional structure. Mostly, they have to build up their own supporting structure in the course of their professional work.

\footnotetext{
${ }^{6}$ Moravcsik was of course working with a traditional, universalistic image of science, in which knowledge always radiates from a metropolis to its various peripheries.

${ }^{7}$ Here Moravcsik remarks that in LDC countries, where science is not yet a "traditional activity," peripheral scientists often lack high degrees of motivation.

${ }^{8}$ See Rajesh Kochar, "Science and Domination: India Before and After Independence," Current Science 76 (1999): 596-601, on a three-stage theory of the origins of modern science in India.
} 
In the hybrid contexts of such peripheral research communities, the context of justification and the contexts of discovery (Reichenbach 1938) can no longer be kept cleanly apart, and the well-known distinction between the context of discovery and context of justification becomes considerably muddled. Contexts of discovery begins to interpenetrate those of justification in such circumstances, for "justification" in these peripheral research communities signifies a range of social and cognitive processes that occur largely outside of that peripheral research community, thereby assuming a delicate dynamic of communication and collaboration between the peripheral community and its metropolitan counterpart. ${ }^{9}$ Peripheral researchers are thus generally faced with a standing set of epistemic constraints. To begin with, they start with a less than critical concentration of researchers at home, which forces them to be dependent on their metropolitan counterparts-not only as inspiration for the subject matters and applications of their research, but also for their validation system, their rewards, and their recognitions of priority. Because they are so dependent, their ability to stick to a line of thinking and nurture it in the face of metropolitan non-acceptance is critically limited. The virtue of tenacity that Feyerabend recommended for budding scientific theories (and thus by extension, to new research communities as well) seems to be rarely available for peripheral researchers.

Thus, in sum, peripheral research communities are characterized by the following general features: 1) an absence of a viable scientific community; 2) an insularity resulting from insufficient access to information or communication; ${ }^{10} 3$ ) a long phase-lag before peripheral researchers become aware of the most significant, hot topics discussed in the metropolis; 4) a weaker institutional infrastructure at home, such as inadequately equipped research laboratories or a less than adequate peer review system; and finally and most importantly, 5) an excessive dependence on science done elsewhere. ${ }^{11}$ While (1) and (4) refer to peripheral researchers' situation at home, (2), (3) and (5) are mostly about their relationship with their corresponding metropolitan community.

Yet, historical evidence indicates that such peripheral communities

\footnotetext{
${ }^{9}$ See David W. Chambers, "Period and Process in Colonial and National Science," in Scientific Colonialism: A Cross-Cultural Comparison, eds. Nathan Reingold and Marc Rothenberg (Washington DC: Smithsonian Institution Press, 1987), 297-321. It is of course presumed in my discussion that justification is not an abstract logical process. Justification, in my view, implies social as well as cognitive processes that are tied to specific locations.

${ }^{10}$ It is in this respect that the World Wide Web can be a friendly tool for a peripheral scientist.

${ }^{11}$ This section depends heavily on Subbiah Arunachalam (1982), and his analysis of the research conditions of a peripheral scientific community.
} 
often succeed, surprisingly, in creating and maintaining a successful culture of scientific research, and can appear progressive-at least for the time being. ${ }^{12}$ In fact, often their very peripheral status acts as their incentive, challenging such communities into singular endeavours of problem-solving in order to gain a foothold of some kind within the reward structure of metropolitan science. This creates, in turn, the beginnings of an autonomous research tradition within their home bases. But notice that in order to be counted as a viable scientific community-let alone a progressive one-a peripheral researcher needs to produce some sort of a striking exemplar that would grant him or her a first entry onto the stage of metropolitan science, achieving some empirical success ${ }^{13}$ for his or her home community. ${ }^{14}$ It is this task that falls on the individual peripheral researcher, who must take up an unsolved issue within metropolitan science and try his or her hand at solving that problem by engaging in some creative scientific reasoning. Thus, the emergence and stabilization of a scientific culture within peripheral communities depends crucially upon their ability to solve some metropolitan problem and create a striking exemplar. But before any exemplars can be created, several other factors must be taken into account: firstly, that smaller scientific community must seize on a problem of maximum epistemic significance-a problem whose solution will be valued at a metropolis. It must then attain a solution for that problem, and finally and most importantly, it must have that solution endorsed by some member(s) of the metropolitan research community. If all these steps go well, then their work provides a foundation upon which a research program can be built at home, where more researchers can participate and contribute, creating an ongoing progressive research tradition. But if this multi-level process breaks down in any of its stages, the peripheral research community does not achieve its aspired state of progressiveness. Thus, progress for such communities depends crucially upon the details of their inter-community dynamic and inter-community collaboration with another, geographically distant but epistemically more privileged, metropolitan community rather than upon the conversations and communications within

\footnotetext{
${ }^{12}$ Japanese microbiology during the early twentieth century and Indian theoretical physics during 1920-1930 are two good examples of such peripheral but progressive scientific communities.

${ }^{13}$ On the notion of empirical success in science, see Miriam Solomon, Social Empiricism (Cambridge, MA: MIT Press, 2001).

${ }^{14}$ 'Home community' refers to more than one's home institution. In a peripheral context, the 'home community' refers to the other institutions and to the researchers who are also engaged in the task of making scientific knowledge. By extension, such a community might also include (some) public intellectuals and technologists who have developed an interest in the fortunes of such scientific endeavours.
} 
their own home community. For the metropolitan researchers, however, precisely the opposite is the case: it is conversation within their own home community that produces a consensus for such researchers. The peripheral researchers, in contrast, must gather their consensus from outside. ${ }^{15}$ This less-than-privileged access to certain epistemic networks and the inability of peripheral researchers to perform a full range of epistemic operations for themselves, which is necessary to generate scientific consensus, creates a non-equality of intellectual authority for such peripheral researchers, placing them within a difficult and derivative form of scientific practice at the peripheries of scientific knowledge.

\section{SCIENTIFIC KNOWLEdge-MAKING WITHIN A PERIPHERAL RESEARCH COMMUNity: THE FEedBack LOOP OF SCIENTIFIC PROGRESS}

If progressiveness can be attained by peripheral researchers, by what mechanism is this progress created and sustained (if only for short-lived periods)? The analysis of the previous section has shown that in peripheral science, there are two aspects to any scientific activity: first, the move to form into a recognizable scientific community, and second, the need to make progress in lockstep with a more privileged metropolitan community from whom the peripheral researcher hopes to obtain a consensus. This metropolitan consensus is thereafter used as a foundation to build a secure tradition of scientific research at home.

These two stages feed into each other in the form of a feedback loop, for success in obtaining a metropolitan consensus clearly enhances success in being a viable scientific community that can undertake more ambitious projects of problem-solving in the future. Historically speaking, a peripheral research community reaches a state of progressiveness when, in a given historical situation, these two stages smoothly mesh into each other, giving rise to a stable tradition at the home base of the peripheral researcher. In the rest of this section, I shall briefly show how such a process briefly happened in one specific periphery-in early twentieth-century colonial India.

The beginnings of modern science in India-like the beginnings of modern science in many other former European colonies-started with an array of field sciences. But during 1910-1930, the flow of Western education, combined with the peaking of national sentiments, produced a new situation. Science, especially basic science, offered an attractive avenue of escape for intelligent minds from the very restricted

\footnotetext{
${ }^{15}$ Whether a peripheral community can generate its own consensus is an interesting question, to which I shall respond below.
} 
opportunities offered by the colonial state. It also allowed a place where those restrictions could be challenged by gaining a ground in pure theoretical knowledge. The result was a short-lived but a flourishing culture in basic sciences that became institutionalized around the metropolitan city of Calcutta, giving rise to many of the country's present-day scientific institutions.

For peripheral scientific researchers, scientific progress unpacks into two stages: first, their proposal of a solution to some current metropolitan research problem, followed by the stage of its validation by some metropolitan researcher(s) that clinches their priority to that solution. The limitations of peripheral researchers stems from the fact that while they can work on a metropolitan problem (and sometimes find a solution), their access to the epistemic processes that validate that solution (thereby establishing their priority) and establish a consensus about their work at the metropolis is a matter that remains largely outside of their grasp. The difficulties of peripheral researchers thus stems from their restricted epistemic access to the knowledge-networks in science, which in turn, also restricts their entry into the reward structure of science. Thus, in a certain sense, the peripheral scientific communities work upon the problems generated by metropolitan scientific communities rather than on problems of their own. Their ability to attain progressiveness is therefore conditional upon someone else performing a number of epistemic operations on their behalf, which makes it more than likely that a peripheral researcher may end up with several failed research programs during the course of his or her professional life. With every successful cycle of the loop in Fig. 1 , a peripheral research community gains more stability that enhances its ability to take on further metropolitan problems, eventually perhaps creating enough exemplars to generate subject matters and applications of its own. When a community thus generates its own subject matters and its own applications-and can produce the necessary consensus for the next cycle of scientific knowledge-making-it gains the status of an autonomous scientific community.

An objection may be raised at this point: can a peripheral researcher generate a consensus based entirely on the resources of his or her own home community? An answer to this question will depend, I think, on the detailed consideration of the stages of that peripheral scientific community's development. When a peripheral scientific community has established a long history of collaboration with a metropolis-having been awarded several episodes of consensus for their work-and has grown into the stage of a sophisticated, urban community with perhaps peripheries of its own, it could at least produce a dissenting view, if not 
a full-fledged consensus, on behalf of its work. ${ }^{16}$ But during an earlier, and a more pioneering stage, such options may not be available for a peripheral researcher, whose work-once rejected by the metropolitan community-frequently suffers complete oblivion in the history of science.

The following diagram describes the process of scientific knowledge-making at the peripheries, the success of which propels a peripheral community towards progressiveness. But alternatively, if the loop fails, it creates a setback for the peripheral researcher.

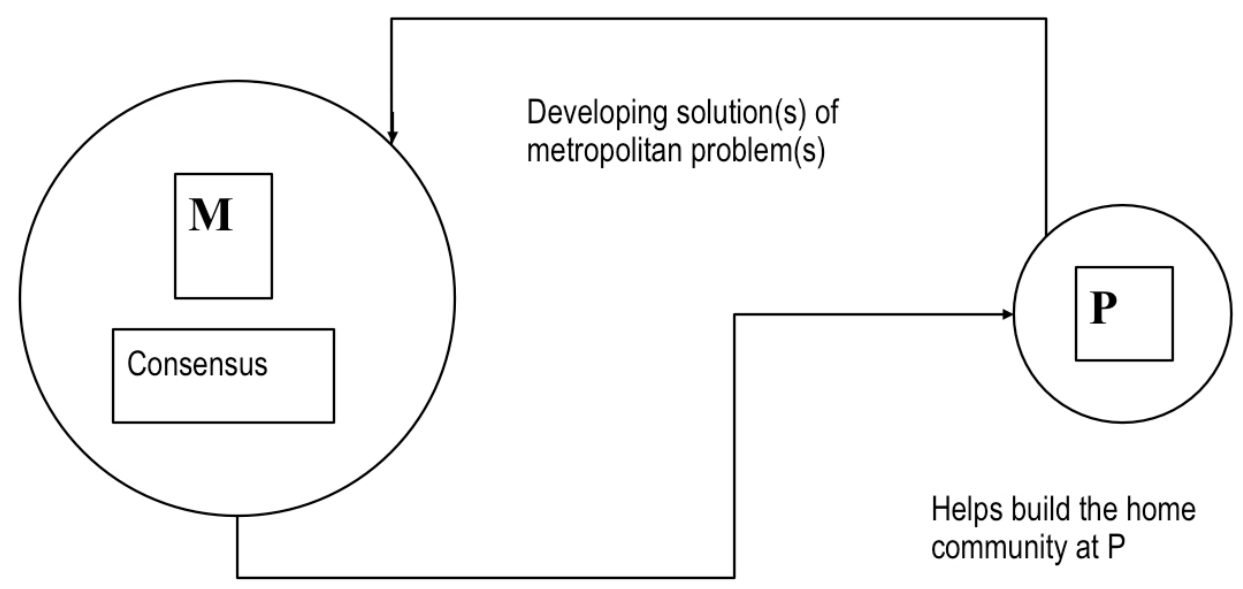

Consensus flows back to $P$ from the metropolis

Figure 1: The feedback loop of scientific progress for a peripheral research community.

When research efforts are distributed between a metropolitan and a peripheral scientific community in a cycle of scientific knowledge-making, which community gets to decide if progress has indeed been made by a peripheral researcher? Historically, it seems that this decision has always been made by the more epistemically privileged metropolitan community.

\section{Progress Made by a Peripheral Scientist: the Story of S.N. Bose and Bose-Einstein Statistics}

The feedback loop that I described above as characteristic of peripheral research communities-the failure of which can set the peripheral

${ }^{16}$ Indeed, C.V. Raman (1888-1970), another Indian physicist, whose research on the crystal lattice structure in diamonds landed him in a sharp controversy with Max Born, may be considered to be an example of this kind of peripheral dissent (to a metropolitan scientific theory). For a detailed analysis of this controversy and its outcome for Raman, see Abha Sur, "Aesthetics, Authority and Control in an Indian Laboratory: The Raman-Born Controversy in Lattice Dynamics," Isis 90:1 (1999): 25-49. 
community back from its course of progressiveness for several decades-is strikingly illustrated in one of the well known episodes of twentieth-century physics-the formulation of Bose-Einstein statistics by a young Indian named Satyendra Nath Bose. ${ }^{17}$ Bose's rigorous derivation of Planck's law in 1924 provided the first non-classical proof of Planck's formula on blackbody radiation. By producing a proof that was free from all classical assumptions of light as a wave, Bose provided a theoretical ground that established Einstein's light-quantum hypothesis beyond all reasonable doubt. His modest four-page paper, titled "Planck's Law and the Light-quantum Hypothesis," published in July 1924 in Zeitschrift fr Physik, provided this solution by means of a simple but remarkable move. ${ }^{18}$ In the course of his elegant proof of Planck's law, Bose simply assumed that the light-quanta in the blackbody radiation field are indistinguishable particles. This implicit assumption (stated nowhere explicitly in his paper) led him to modify Boltzmann's statistics in a novel manner. By mentally modelling the radiation field as a grid of phase spaces, Bose in effect transformed the field into a collection of phase space cells within which the light quanta could be accommodated. He then calculated the equilibrium of this revised system by simply calculating in how many ways those cells could be populated by (indistinguishable) particles, instead of counting how many individual particles could occupy a given number of cells (the old Boltzmannian statistics). This neat reversal introduced what is called the indistinguishability hypothesis in quantum mechanics, laying the foundation for a new kind of statistics. With Einstein's endorsement of Bose's idea and his application of Bose's counting procedure to the theory of monoatomic gases, a new quantum statistics was born, and a quantum theory based on the indistinguishability hypothesis ushered in a new revolution in physics. Yet, astonishing though it seems, Bose did not take any part into this larger transformation, in spite of producing one of its fundamental early results. In fact, shortly after 1926 he moved altogether away from radiation theory, thus raising the suspicion among his European colleagues that he had become a drop-out from science. How can we make sense of Bose's initial involvement-and his later withdrawal-from a topic that once so intensely interested him?

Without Einstein's personal support-it was he who communicated Bose's first paper to Zeitschrift fr Physik, translating it into German-Bose might never have published his result, for he had already been rejected

${ }^{17}$ Or S. N. Bose, as he was known to his colleagues in the West.

${ }^{18}$ Bose's first paper was translated by Einstein and submitted to Zeitschrift fr Physik on 2 July 1924. For an English translation of this paper, see Santimoy Chatterjee, ed. S.N. Bose: the Man and His Work, vol. 1. (Calcutta: S.N. Bose Centre for Basic Sciences, 1994): 100-103. 
by the Philosophical Magazine. Born in 1853 in the colonial British city of Calcutta, Bose was among the first generation of Indian researchers who had the objective of setting up a new tradition of scientific research at their home base. As peripheral scientists working from a distant British colony, this small group of young men of which Bose was a part, began their research by taking an interest in the 'new science' that was then sweeping Europe. ${ }^{19}$ It is in the course of their encounter with this 'new science' that Bose came upon the blackbody radiation problem which was then confronting the metropolitan community in Europe. Planck's law had provided the experimentally correct result, but it was still riddled with theoretical contradictions. Bose's choice of subject matter thus came from his grasp of this metropolitan impasse. By reasoning consistently on the basis of a mental model that saw radiation as a gas of light quanta-his very isolation cutting him off from the thought patterns of other European physicists-Bose was able to carry out a reversal in established thinking. When his solution was rejected by the Philosophical Magazine, Bose sent his paper to Einstein instead, and Einstein's quick endorsement of his results-and its speedy extension to the problem of monoatomic gases-created the first metropolitan consensus for Bose and his small scientific community in India.

Unfortunately, the feedback loop that worked flawlessly the first time failed on Bose's second, and a more ambitious, attempt. Bose wrote a second paper immediately after the first on the topic of the interaction of matter and radiation, and as usual, he sent it to Einstein. In an ambitious two-part project, in this paper Bose sought to derive the general conditions for the statistical equilibrium of a system that contained both matter and radiation but was independent of any special assumptions about the nature of those radiative processes. ${ }^{20}$ Hoping to build upon Einstein's work and remove all arbitrary assumptions from that work (just as he had done the first time), Bose rejected Einstein's special assumptions about there being two kinds of radiative processes-spontaneous and induced-by means of which an atom of higher energy shifts down to a lower energy level. Instead, he claimed that the transition from a higher energy state to a lower energy state could be explained without using an additional hypothesis, such as spontaneous transitions. A spontaneous transition, such as radioactivity, could be explained, Bose claimed, more

\footnotetext{
${ }^{19}$ In order to provide themselves with some key texts for this 'new' science, Bose and his colleague, M.N. Saha, translated Einstein's papers on General Relativity from German into English, thereby producing the first English translation of those papers.

${ }^{20}$ The title of Bose's second paper in English translation is: "Thermal Equilibrium in the Radiation Field in the Presence of Matter," submitted to Zeitschrift fr Physik on 7 July 1924 by Einstein. For an English translation of the second paper, see S.N. Bose: the Man and His Work, vol 1, 114-21.
} 
straightforwardly as having arisen out of the statistical properties of the radiation field itself, consistent with all equilibrium conditions.

This time Bose failed to wrest a consensus for his novel idea of a unified radiative process. Einstein did communicate Bose's paper to the same journal, but he appended a strongly critical note to the paper and rejected Bose's ideas in personal communication. Thus Bose's ambitious project of developing a research program built on the notion of a unified radiative process was abandoned in the face of Einstein's non-acceptance. The gist of Bose's idea came back later in Dirac's method of second quantization, but by then it was too late to resurrect either Bose or his views on radiation. ${ }^{21}$ Faced with opposition from the greatest metropolitan authority in science of his time-Einstein-Bose considered it prudent to abandon the topic of radiation theory altogether and move away to different, and to more experimental fields of science, such as crystallography and chemistry. Thus his second paper on radiation theory lay forgotten among the pages of Zeitschrift fr Physik, a precursor to the later quantum theory but nothing more. For a peripheral researcher, focused intently upon the task of developing an independent culture of scientific research at home, Bose's most prudent move was to shift his focus of research to an area where a consensus from metropolitan community could be more easily obtained. Thus, he applied himself to chemistry and crystallography, in which one of his younger colleagues, K. S. Krishnan, later did well-known work, initiating a tradition of crystallographic research at Dhaka University (in present-day Bangladesh). But this was not done under Bose's own name.

Thus, Bose's remarkable initial success in proving Planck's law and his later disappointment over his second paper in radiation theory shows us how a colonial researcher who seeks to make scientific knowledge from a periphery builds a network with his or her metropolitan scientific community, and how the fragile feedback loop-of which the colonial researcher is a part-can create a progressive scientific tradition at the peripheries by repeated iterations. But conversely, if the loop fails, it lands the peripheral researcher with a failed research program, which clearly happened to Bose with his second paper.

What exactly did Bose contribute to the peripheral Indian community as it existed during the decades of 1920s? Since his own metropolitan success consisted of a brilliant but a brief episode, Bose personally was not able to create many iterated loops of knowledge. My argument, however, is that he succeeded in creating a path for such future iterations. After Bose, other peripheral researchers from his home community could

\footnotetext{
${ }^{21}$ For a detailed discussion of Bose's second paper, and whether his proposals were indeed precursors of the new quantum theory, see Partho Ghosh, "Bose-Statistics: A Historical Perspective," in S.N. Bose: the Man and His Work, vol. 1. 35-67.
} 
join in science and build upon the exemplars left by their previous generations, eventually creating a stronger network of scientific knowledge at the periphery. In this sense, Bose acted as a pioneer for the nascent scientific community in India, giving it a template of what it is to be a peripheral scientist from such a location.

\title{
IV. CONCLUSION
}

One of the consequences of this analysis is that we see a clear case of persistent asymmetry in intellectual authority arising between the peripheral and metropolitan research communities, especially in those cases when a scientific controversy arises between the two. This persistent inequality differentially influences the output of knowledge by a peripheral community and thus influences its subsequent success at creating and sustaining an autonomous and progressive research tradition in its home grounds. This result is contrary to several analyses of scientific knowledge developed in the recent times from a social-epistemological point of view, such as that of Longino (1990, 78-79), who maintains that an equality of intellectual authority is the norm in scientific communication among different research communities. But if the history of the scientific endeavours of colonial and peripheral scientific communities are fully taken into account, such claims may turn out to be-on closer scrutiny-more like cases of hopeful fiction. Good science, however, may yet be born of those interactions that are characterized by this kind of prominent asymmetry.

\author{
DEEPANWITA DASGUPTA \\ Department of Philosophy \\ 831 Heller Hall \\ University of Minnesota \\ Minneapolis, MN 55455 \\ dasgu007@umn.edu \\ Ph: 612-331-0108 \\ Fax: 612-626-8380
}

\section{REFERENCES}

Arunachalam, Subbiah. 1982. Peripherality in Science: What Should be Done to Help Peripheral Science Get Assimilated into Mainstream Science. Technical Report, New Delhi: Publications and Information Directorate.

Bird, Alexander. 2007. What is Scientific Progress? Nous, 42(2): 92-117. 
Chambers, David Wade. 1987. Period and Process in Colonial and National Science. In Scientific Colonialism: A Cross- Cultural Comparison, eds. Nathan Reingold and Marc Rothenberg, 297-321. Washington DC: Smithsonian Institution Press.

Chatterjee, Santimoy, ed. S.N. Bose: The Man and His Work. Vol. 1. Calcutta: S.N. Bose Center for Basic Sciences.

Feyerabend, Paul. 1988. Against Method. London; New York: Verso.

Ghosh, Partho. 1994. Bose-Statistics: A Historical Perspective. In S.N.Bose: the Man and His Work, Vol. 1. ed. Santimoy Chatterjee, 35-67. Calcutta: S.N. Bose Centre for Basic Sciences.

Kitcher, Philip.1993. Advancement of Science: Science without Legend, Objectivity without Illusions. Oxford: Oxford University Press.

Kuhn, Thomas.1962. The Structure of Scientific Revolutions. Chicago: University of Chicago Press.

Kochar, Rajesh. 1999. Science and Domination: India before and after Independence. Current Science 76 (Feb): 596-601.

Lakatos, Imre and Alan Musgrave, eds. 1970. Criticism and Growth of Knowledge. Cambridge, MA: Cambridge University Press.

Laudan, Larry. 1977. Progress and Its Problems: Towards a Theory of Scientific Growth. Berkeley: University of California Press.

Longino, Helen. 1990. Science as Social Knowledge. Princeton; NJ: Princeton University Press.

Moravcsik, Michael. 1978. Do Less Developed Countries Have a Special Science of Their Own? Interciencia 3 (Jan-Feb): 8-13.

Niiniluoto, Ilkka.1984. Is Science Progressive? Dordrecht: D. Reidel.

Popper, Karl. 1963. Conjectures and Refutations: The Growth in Scientific Knowledge. London: Hutchinson.

Solomon, Miriam. 2001. Social Empiricism. Cambridge, MA: MIT Press.

Reichenbach, Hans. 1938. Experience and Prediction. Chicago: University of Chicago Press.

Sur, Abha. 1999. Aesthetics, Authority and Control in an Indian Laboratory: The Raman-Born Controversy in Lattice Dynamics. Isis, 90 (1): 25-49.

Traweek, Sharon. 1988. Beamtimes and Lifetimes: the World of High Energy Physicists. Cambridge, MA: Harvard University Press. 